\title{
Vision-related Quality of Life in Malaysian Children with Threshold and Prethreshold Retinopathy of Prematurity
}

\author{
Chong Wern-Yih ${ }^{1,2,3}$, Qamarruddin Fazilawati ${ }^{3}$, Jamalia Rahmat ${ }^{4}$, Ismail Shatriah ${ }^{1,2}$ \\ ${ }^{1}$ Department of Ophthalmology and Visual Science, School of Medical Sciences, Universiti Sains Malaysia, Kubang Kerian, Malaysia \\ ${ }^{2}$ Ophthalmology Clinic, Hospital Universiti Sains Malaysia, Kubang Kerian, Malaysia \\ ${ }^{3}$ Department of Ophthalmology, Hospital Tengku Ampuan Rahimah Klang, Klang, Malaysia \\ ${ }^{4}$ Department of Ophthalmology, Hospital Kuala Lumpur, Kuala Lumpur, Malaysia
}

\begin{abstract}
Purpose: The prevalence of retinopathy of prematurity (ROP) is higher in developing countries compared to developed countries. There is limited data on vision-related quality of life (VRQoL) among children with severe type of ROP in developing countries. This study evaluated the influence of threshold and prethreshold ROP on VRQoL in Malaysian children.

Methods: Multicenter prospective cross-sectional study conducted in three tertiary hospitals in 2018 to 2019 . Children less than 7 years old with previous ROP diagnosis were recruited. Patients with systemic comorbidities that affected vision or daily activities were excluded. A parent or guardian completed the Children's Visual Function Questionnaire (CVFQ) for the assessment of child's general health, general vision, competence, personality, family impact, and treatment difficulty.

Results: Eight were categorized with threshold ROP, 16 with high-risk prethreshold ROP, and 26 with low-risk prethreshold ROP. Fifty age-matched controls were also included. Mean visual acuity in logarithm of the minimum angle of resolution was 0.46 in the threshold, 0.08 in high-risk prethreshold, and 0.01 in low-risk prethreshold subgroups. Threshold ROP was associated with myopia and strabismus, and associated with poor visual acuity compared to prethreshold ROP. Mean total CVFQ score was significantly lower in the ROP group $(p<0.001)$ compared to the control group. Mean score and all mean subscale scores were significantly lower in the threshold subgroup compared to high-risk and low-risk prethreshold subgroups, with lowest subscale scores on general vision and general health. There was significant association between gestational age, visual acuity of the better eye, family income, and VRQoL $(p<0.05)$.

Conclusions: ROP was associated with lower VRQoL in children born prematurely in Malaysia. The threshold ROP group is the most affected. General vision and health domains are their main difficulties encountered. Gestational age, visual acuity of the better eye, and family income affects the VRQoL.
\end{abstract}

Key Words: Children's Visual Function Questionnaire, High-risk prethreshold, Low-risk prethreshold, Retinopathy of prematurity, Threshold group

Corresponding Author: Ismail Shatriah, MD. Department of Ophthalmology and Visual Science, School of Medical Sciences, Universiti Sains Malaysia, Kubang Kerian 16150, Malaysia. Tel: 60-9767-6362, Fax: 609765-3370,E-mail: shatriah@usm.my
Retinopathy of prematurity (ROP) is a potentially blinding disease that affects immature retinal vasculature in the eyes of premature low birth weight babies. It is a leading cause of childhood blindness, accounting for up to 
$12 \%$ of pediatric vision impairment cases worldwide in developed countries [1-3]. However, the prevalence of ROP among premature children varies markedly by region, averaging $21 \%$ in Asian countries, $29.2 \%$ to $36.6 \%$ among all developed nation, and $11.9 \%$ to $58.6 \%$ among all developing countries [4-10].

Threshold ROP was defined as ROP of more than 5 contiguous or 8 cumulative clock hours of stage 3 plus ROP in zone 1 or zone 2. While prethreshold ROP was considered as any zone 1 ROP less than threshold, zone 2 stage 2 with plus, zone 2 stage 3 without plus, or zone 2 stage 3 with plus but less than 5 contiguous or less than 8 cumulative clock hours of ROP. Diode laser treatment is the standard treatment for premature babies with threshold ROP. The proportion of premature babies in developing countries that required laser treatment for ROP also varies; for instance, while only $6.5 \%$ to $9.3 \%$ in Taiwan, and $11.5 \%$ in South Korea, $21 \%$ in Indonesia, $25.4 \%$ in India, and 32.9\% in Malaysia require laser treatment [5-8,10].

The majority of children with laser treated threshold ROP have favorable anatomical and visual outcomes. Vision-related quality of life (VRQoL) in children with ROP has been assessed in Brazil and India [11,12], but there are little data from developing countries of South or East Asia according to a recent PubMed search. Such information is critical because more severe types of ROP have been observed in developing countries with limited health resources for neonatal care $[13,14]$. Further, these data could aid in more efficient allocation of resources [14].

Children's Visual Function Questionnaire (CVFQ) is a validated and specific instrument designed to measure VRQoL in children up to 7 years of age by parent or caregiver report [15]. Our study aimed to compare the mean total and mean subscales scores (general health, general vision, competence, personality, family impact, and treatment) among children with ROP stratified according to disease severity (threshold, high-risk prethreshold, and low-risk prethreshold).

\section{Materials and Methods}

This was a multicenter prospective cross-sectional study conducted in three tertiary hospitals with pediatric ophthalmology services from December 2018 to November 2019: Hospital Universiti Sains Malaysia, Hospital Tengku
Ampuan Rahimah, and Hospital Kuala Lumpur. The study was approved by the Research and Ethics Committee of School of Medical Sciences, Universiti Sains Malaysia (No. USM/JEPeM/17110578) and the National Medical Research Registry of Ministry of Health, Malaysia (No. NMRR-17391-39410). All study protocols were conducted in adherence with the principles of the Helsinki Declaration and Resolution 196/96. Informed consent was obtained from each parent or guardian before participation.

The children and parents or guardians were selected through simple random sampling from the Ophthalmology Pediatric Registry of the participating hospitals. Children aged 7 years old or less with previous history of ROP and born at 32 weeks of gestation or earlier were eligible for the study. The control group was composed of children up to 7 years old and born full-term. The exclusion criteria for children were chronic encephalopathy, hydrocephalus, microcephaly, macrocephaly, central nervous system malformation, intracranial hemorrhage, cerebral visual impairment, and intellectual disability. Children with a history of glaucoma, corneal opacity, nystagmus, microcornea, or amblyopia were also excluded. One parent or guardian of these children participated in this study. Parents or guardians were excluded (with the child) if they refused to answer the questionnaire or had poor English proficiency.

The study was conducted in two phases. In the first phase, the children were recruited according to the final diagnosis of ROP based on the International Classification of Retinopathy of Prematurity [16]. In the second phase, the recruited children with a history of ROP were divided into three subgroups, threshold ROP, high-risk prethreshold ROP, and low-risk prethreshold ROP, as defined by the Cryo-ROP study and Early Treatment Trial of ROP [17,18].

Birth history, gestational age, diagnosis of ROP, and treatment received were obtained from medical records. Educational level and family income were obtained from the parent or guardian. All children in the ROP and control groups had visual acuity and complete ophthalmic assessments. Bilateral visual acuity measures were converted to logarithm of the minimum angle of resolution. Cycloplegic refraction measures were performed by a trained optometrist.

There are two versions of the CVFQ, one for children under 3 years of age and another for children 3 to 7 years of age [15]. The questionnaire for younger children contains 35 items and for older children 40 items on topics re- 
lated to visual function and VRQoL. Each version has six subscales evaluating general health, general vision, competence, personality, family impact, and treatment difficulty. Subscale scores range from 0 (worst) to 1 (best). The mean score for each subscale was calculated from all subscale questions and the mean total score was calculated as the sum of mean subscale scores. Unanswered questions and "not applicable" responses were omitted from the mean score calculations.

The questionnaire was completed by one parent or guardian during a 30-minute private interview in an enclosed room. The parent or guardian was allowed to ask for clarification on specific items. Instructions were also provided on the first page of the questionnaire. The parent or guardian were instructed to choose a response option for each question or omit the question if the option was not

Table 1. Demographic and clinical characteristics

\begin{tabular}{|c|c|c|c|}
\hline Characteristic & Children with ROP & Control group & $p$-value \\
\hline Gestational age (wk) & $29.6 \pm 0.29$ & $38.3 \pm 0.21$ & $<0.001^{*}$ \\
\hline Birth weight (kg) & $1.53 \pm 0.05$ & $2.84 \pm 0.05$ & $<0.001^{*}$ \\
\hline Current age (yr) & $4.88 \pm 0.25$ & $4.26 \pm 0.27$ & 0.326 \\
\hline Sex & & & NA \\
\hline Male & $23(46)$ & $24(48)$ & \\
\hline Female & $27(54)$ & $26(52)$ & \\
\hline Parents'/guardians' education level & & & NA \\
\hline Primary & $2(4)$ & $1(2)$ & \\
\hline Secondary & $23(46)$ & $10(20)$ & \\
\hline Tertiary & $25(50)$ & $39(78)$ & \\
\hline Family income per month & & & NA \\
\hline Less than RM 1,000 & $6(12)$ & $0(0)$ & \\
\hline RM 1,000-2,999 & $16(32)$ & $17(34)$ & \\
\hline RM 3,000-9,999 & $25(50)$ & $14(28)$ & \\
\hline More than RM 10,000 & $3(6)$ & $19(38)$ & \\
\hline Treatment & & NA & NA \\
\hline Conservative & $26(52)$ & & \\
\hline Laser PRP & $20(40)$ & & \\
\hline Trans pars plana vitrectomy & $3(6)$ & & \\
\hline Laser PRP and intravitreal anti-VEGF & $1(2)$ & & \\
\hline Successful anatomical outcome & $50(100)$ & NA & NA \\
\hline \multicolumn{4}{|l|}{ Current visual acuity (logMAR) } \\
\hline Right eye & $0.10 \pm 0.22$ & $0.01 \pm 0.05$ & $<0.001^{*}$ \\
\hline Left eye & $0.15 \pm 0.32$ & $0.01 \pm 0.03$ & $<0.001^{*}$ \\
\hline Ocular diseases & & & $<0.001^{* \dagger}$ \\
\hline None & $21(42)$ & $43(86)$ & \\
\hline Myopia & $17(34)$ & $7(14)$ & \\
\hline Strabismus & $3(6)$ & $0(0)$ & \\
\hline Myopia and strabismus & $9(18)$ & $0(0)$ & \\
\hline
\end{tabular}

Values are presented as mean \pm standard deviation or number (\%); independent $t$-test $(p<0.05$ is significant).

$\mathrm{ROP}=$ retinopathy of prematurity; NA = not available; $\mathrm{RM}=$ Ringgit Malaysia; $\mathrm{PRP}=$ panretinal photocoagulation; VEGF $=\mathrm{vascular}$ endothelial growth factor; $\log \mathrm{MAR}=\operatorname{logarithm}$ of the minimum angle of resolution.

${ }^{*}$ Statistically significant value; ${ }^{\dagger}$ Fisher exact test $(p<0.05$ is significant). 
available and/or refused to be answered.

All data were collected and analyzed using the IBM SPSS ver. 24 (IBM Corp., Armonk, NY, USA). All numerical data are expressed as mean \pm standard deviation, median \pm interquartile range, and categorical data as frequency (\%). Mean total CVFQ scores and all subscale scores were compared between ROP and control groups by independent samples $t$-test and among ROP subgroups by oneway ANOVA. Categorical variables were compared between group and subgroups by Fisher exact test. We used a multiple linear regression model based on the mean total CVFQ score to determine the effect of ROP and other variables (i.e., gestational age, current age, visual acuity of the better eye, family income). A $p$-value less than 0.05 was considered statistically significant.

\section{Results}

Fifty children with ROP and 50 healthy control children were recruited. Table 1 summarizes demographic and baseline clinical information for both groups. Mean age and sex ratio did not differ between groups. As expected, mean gestational age and birth weight were lower among children with ROP. In addition, the proportion of parents or guardian receiving only primary or secondary education was higher in the ROP group, and there were more low-income families in the ROP group.

Forty percent of the children in the ROP group had received panretinal photocoagulation, $6 \%$ pars plana vitrectomy, and $2 \%$ had a combination of panretinal photocoagulation and intravitreal injection of antivascular endothelial growth factor. The remaining ROP group children improved spontaneously without intervention. There was a significant difference in mean visual acuity for both eyes between ROP and control group children $(p<0.001)$. The prevalence of myopia was significantly higher in the ROP group. There were no cases of retinal detachment in either group.

Table 2 presents the distribution of threshold, high-risk prethreshold, and low-risk prethreshold children in the ROP group, and compares the clinical features among these subgroups. None had aggressive posterior ROP. There were significant differences in mean gestational age,

Table 2. Clinical characteristics of ROP subgroups

\begin{tabular}{|c|c|c|c|c|c|}
\hline Characteristic & $\begin{array}{l}\text { Children with } \\
\text { threshold ROP }\end{array}$ & $\begin{array}{c}\text { High-risk } \\
\text { prethreshold ROP }\end{array}$ & $\begin{array}{c}\text { Low-risk } \\
\text { prethreshold ROP }\end{array}$ & $F$-statistic (df) & $p$-value \\
\hline Gestational age (wk) & $26.5 \pm 0.76$ & $29.31 \pm 1.78$ & $30.73 \pm 1.31$ & $27.70(2,47)$ & $<0.001^{*}$ \\
\hline Birth weight $(\mathrm{kg})$ & $1.06 \pm 0.14$ & $1.43 \pm 0.21$ & $1.73 \pm 0.29$ & $23.67(2,47)$ & $<0.001^{*}$ \\
\hline Treatment & & & & - & $<0.001^{\dagger}$ \\
\hline Conservative & $0(0)$ & $0(0)$ & $26(100)$ & & \\
\hline Laser PRP & $4(50.0)$ & $16(100)$ & $0(0)$ & & \\
\hline Trans pars plana vitrectomy & $3(37.5)$ & $0(0)$ & $0(0)$ & & \\
\hline Laser PRP and intravitreal anti-VEGF & $1(12.5)$ & $0(0)$ & $0(0)$ & & \\
\hline \multicolumn{6}{|l|}{ Visual acuity $(\log M A R)$} \\
\hline Right eye & $0.46 \pm 0.37$ & $0.08 \pm 0.1$ & $0.01 \pm 0.04$ & $26.16(2,47)$ & $<0.001^{*}$ \\
\hline Left eye & $0.61 \pm 0.62$ & $0.11 \pm 0.1$ & $0.04 \pm 0.08$ & $16.26(2,47)$ & $<0.001^{*}$ \\
\hline Ocular diseases & & & & - & $<0.001^{\dagger}$ \\
\hline None & $0(0)$ & $2(12.5)$ & $19(73.1)$ & & \\
\hline Myopia & $1(12.5)$ & $11(68.8)$ & $5(19.2)$ & & \\
\hline Strabismus & $1(12.5)$ & $1(6.3)$ & $1(3.8)$ & & \\
\hline Myopia and strabismus & $6(75.0)$ & $2(12.5)$ & $1(3.8)$ & & \\
\hline
\end{tabular}

Values are presented as mean \pm standard deviation, number (range), or number $(\%)$; one-way ANOVA $(p<0.05$ is significant). $\mathrm{ROP}=$ retinopathy of prematurity; $\mathrm{df}=$ degree of freedom; PRP = panretinal photocoagulation; VEGF $=$ vascular endothelial growth factor; $\log \mathrm{MAR}=\log$ arithm of the minimum angle of resolution.

${ }^{*}$ Statistically significant value; ${ }^{\dagger}$ Fisher exact text $(p<0.05$ is significant). 
mean birth weight, and treatments received among subgroups $(p<0.001)$. Mean current visual acuity was significantly poorer in the threshold subgroup compared to both prethreshold groups $(p<0.001)$. The prevalence of myopia was greater in the high-risk prethreshold subgroup than the threshold or low-risk prethreshold group, while strabismus with myopia was highest in the threshold group.

Mean total CVFQ scores and all subscale scores were significantly lower in the ROP group than the control group $(p<0.01)$ (Table 3). Mean total score and subscale scores also differed significantly among the three ROP subgroups $(p<0.001)$ with lowest scores in the threshold subgroup and highest scores in the low-risk prethreshold subgroup (Table 4).

We found significant association between gestational age, visual acuity of the better eye, family income, and mean total score of CVFQ $(p<0.05)$. Table 5 shows the $p$-values for each of these variables.

\section{Discussion}

Asian infants were reported to develop severe ROP at higher rates than Caucasian infants [19]. In a previous study from Malaysia, $50.1 \%$ of premature babies with ROP were classified threshold disease at one of the participating institutions, including 39.3\% with stage 3, 3.6\% with stage 4 , and $7.2 \%$ with stage 5 disease [20]. Moreover, $17.4 \%$ of the students attending 24 special schools in Malaysia were blind or severely visual impaired as a result of ROP [21].

Our study further documented significantly reduced VRQoL among Malaysian children with ROP according to the CVFQ. This finding is in accord with previous studies in Brazil and India [11,12]. Messa et al. [11] used a different

Table 3. Children's Visual Function Questionnaire total and subscale scores for ROP and control groups

\begin{tabular}{|c|c|c|c|c|}
\hline Subscale & Mean score \pm SD & Mean difference $(95 \% \mathrm{CI})$ & $t$-statistic (df) & $p$-value \\
\hline Mean total score & & $0.32(0.27-0.36)$ & $14.96(98)$ & $<0.001^{*}$ \\
\hline ROP & $0.67 \pm 0.15$ & & & \\
\hline Normal & $0.98 \pm 0.02$ & & & \\
\hline General health & & $0.36(0.29-0.43)$ & $9.62(58)$ & $<0.001^{*}$ \\
\hline ROP & $0.62 \pm 0.25$ & & & \\
\hline Normal & $0.98 \pm 0.08$ & & & \\
\hline General vision & & $0.27(0.20-0.34)$ & $7.99(98)$ & $<0.001^{*}$ \\
\hline ROP & $0.73 \pm 0.24$ & & & \\
\hline Normal & $0.99 \pm 0.04$ & & & \\
\hline Competence & & $0.30(0.25-0.35)$ & $11.40(56)$ & $<0.001^{*}$ \\
\hline ROP & $0.67 \pm 0.18$ & & & \\
\hline Normal & $0.97 \pm 0.05$ & & & \\
\hline Personality & & $0.35(0.31-0.39)$ & $17.82(98)$ & $<0.001^{*}$ \\
\hline ROP & $0.64 \pm 0.14$ & & & \\
\hline Normal & $0.99 \pm 0.03$ & & & \\
\hline Family impact & & $0.27(0.23-0.32)$ & $11.59(71)$ & $<0.001^{*}$ \\
\hline ROP & $0.69 \pm 0.15$ & & & \\
\hline Normal & $0.96 \pm 0.07$ & & & \\
\hline Treatment & & $0.74(0.64-0.84)$ & $14.78(98)$ & $<0.001^{*}$ \\
\hline ROP & $0.26 \pm 0.35$ & & & \\
\hline Normal & $1.00 \pm 0.00$ & & & \\
\hline
\end{tabular}

Independent $t$-test $(p<0.05$ is significant).

$\mathrm{ROP}=$ retinopathy of prematurity; $\mathrm{SD}=$ standard deviation; $\mathrm{CI}=$ confidence interval; $\mathrm{df}=$ degree of freedom .

*Statistically significant value. 
classification system for disease severity (stages 1-3 and stages 4-5), while Kesarwani et al. [12] reported the outcome according to zone (zone I and II) and segregated patients according to age less than 3 years and age 3 years or more.

The mean subscale scores were also consistent with Messa et al. [11]. Mean score in our study ranged from 0.62 to 0.73 among the ROP group but was substantially lower for the items evaluating the effects of treatment on the child and family (mean score, $0.26 \pm 0.35$ ). These scores suggest that parents experienced difficulty with treatment (i.e., glasses and eye patches) for the children with ROP. Nevertheless, we were unable to compare treatment subscale with Messa et al. [11] because they did not analyze this component. In contrast, the mean subscale scores between the two different age groups were higher than that of reported by Kesarwani et al. [12].

General vision was the main CVFQ subscale impacted by disease severity, as mean subscale score was markedly lower in children with threshold disease compared to high-

Table 4. Children's Visual Function Questionnaire total and subscale scores for ROP subgroups

\begin{tabular}{|c|c|c|c|}
\hline Subscale & Mean score \pm SD & $F$-statistic (df) & $p$-value \\
\hline Mean total score & & $39.72(2,47)$ & $<0.001^{*}$ \\
\hline Threshold ROP & $0.47 \pm 0.12$ & & \\
\hline High-risk prethreshold & $0.68 \pm 0.09$ & & \\
\hline Low-risk prethreshold & $0.81 \pm 0.09$ & & \\
\hline General health & & $13.97(2,47)$ & $<0.001^{*}$ \\
\hline Threshold ROP & $0.34 \pm 0.19$ & & \\
\hline High-risk prethreshold & $0.53 \pm 0.24$ & & \\
\hline Low-risk prethreshold & $0.75 \pm 0.19$ & & \\
\hline General vision & & $66.16(2,47)$ & $<0.001^{*}$ \\
\hline Threshold ROP & $0.25 \pm 0.26$ & & \\
\hline High-risk prethreshold & $0.70 \pm 0.10$ & & \\
\hline Low-risk prethreshold & $0.87 \pm 0.10$ & & \\
\hline Competence & & $9.66(2,47)$ & $<0.001^{*}$ \\
\hline Threshold ROP & $0.47 \pm 0.14$ & & \\
\hline High-risk prethreshold & $0.62 \pm 0.18$ & & \\
\hline Low-risk prethreshold & $0.75 \pm 0.16$ & & \\
\hline Personality & & $4.69(2,47)$ & $<0.001^{*}$ \\
\hline Threshold ROP & $0.52 \pm 0.13$ & & \\
\hline High-risk prethreshold & $0.63 \pm 0.14$ & & \\
\hline Low-risk prethreshold & $0.68 \pm 0.12$ & & \\
\hline Family impact & & $15.95(2,47)$ & $<0.001^{*}$ \\
\hline Threshold ROP & $0.57 \pm 0.06$ & & \\
\hline High-risk prethreshold & $0.60 \pm 0.14$ & & \\
\hline Low-risk prethreshold & $0.78 \pm 0.11$ & & \\
\hline Treatment & & $31.20(2,47)$ & $<0.001^{*}$ \\
\hline Threshold ROP & $0.65 \pm 0.30$ & & \\
\hline High-risk prethreshold & $1.00 \pm 0.00$ & & \\
\hline Low-risk prethreshold & $1.00 \pm 0.00$ & & \\
\hline
\end{tabular}

One-way ANOVA $(p<0.05$ is significant $)$.

$\mathrm{ROP}=$ retinopathy of prematurity; $\mathrm{SD}=$ standard deviation; $\mathrm{df}=$ degree of freedom.

${ }^{*}$ Statistically significant value. 
risk and low-risk prethreshold disease. Myopia and strabismus were the main causes of this difference, observed in $100 \%$ of the threshold group but only $26.8 \%$ of the low-risk prethreshold group. In contrast, Kesarwani et al. [12] reported more favorable general vision subscale scores in zone I ROP (mean score, $0.90 \pm 0.20$ ) and zone II ROP (mean score, $0.98 \pm 0.12$ ), but $88.9 \%$ of their patients had good visual acuity (logarithm of the minimum angle of resolution $\geq 0.3$ ).

Surprisingly, general health was the second most severely affected subscale reported by parents despite excluding candidates with general health issues and chronic systemic illness during recruitment. We suggest that poorer visual acuity with limits participation in sports and other outdoor activities at school and home, which both denies children the associated health benefits. This may be a source of anxiety for the parents as reported by Ozyurt et al. [22] in Turkey. Low educational level and poor socioeconomic status were reported to aggravate the stress experienced by mothers handled children with prematurity [23].

The remaining subscales (competence, family impact, personality, and treatment) exhibited a similar pattern. Vision-related daily activities were less affected in the lowrisk prethreshold subgroup while the threshold group was the most affected $(p<0.001)$, fairly consistent with Messa et al. [11] who compared stages 1-3 ROP to stages 4-5 ROP. However, the children with ROP in our study had poorer visual acuity overall, which could explain why competence, personality, family impact, and treatment subscales were also poorer in the threshold subgroup. The treatment subscale in our study had the least impact on VRQoL among children with ROP. This is possibly because all the centers involved in this study are government hospitals providing low-cost treatment and consultation.

Premature babies achieved lower intellectual, language, memory, and attention abilities compared to term born ba- bies, which contributed to the lower subscales scores [24]. It can be explained by neurophysiological hypothesis postulated by Scher et al. [25], that term babies have fivefold increase in white matter volume and better development, while preterm babies are more vulnerable to remote injury in their brain; thus, it leads to neurocognitive and behavioral deficits.

We observed a significant association between gestational age, visual acuity of the better eye and family income with VRQoL. This data can be justified that by the fact that extremely younger babies require frequent visits to the clinics for ocular examinations especially during the first 2 months of life. Family with poorer outcome will be affected more compared to those with better outcome. Children with ROP who have better visual acuity enjoy a better quality of life.

Differences in VRQoL have been reported between developing and developed countries. Kesarwani et al. [12] reported better competence and personality subscale scores among patients with zone I ROP compared to zone II ROP, while Birch et al. [15] observed significantly better competence, personality, and family impact among American children with ROP and normal visual acuity. Additional studies on VRQoL are warranted in developing countries with more limited treatments, rehabilitation resources, and public services for visually impaired. The major limitation of the present study was absence of the group with similar morbidity. Inclusion of this group will give a better idea and understanding in the future research.

In conclusion, children with threshold ROP were the most severely affected. General vision and health are their main difficulties encountered. These issues merit attention from ophthalmologists, neonatologists, and health policy makers in a developing country.

Table 5. Estimates of adjusted multiple linear regression model of mean total score of Children's Visual Function Questionnaire

\begin{tabular}{lccc}
\hline Model & Coefficient & Standard error & $p$-value \\
\hline Gestational age & 0.024 & 0.002 & $<0.001^{\dagger}$ \\
Current age & -0.003 & 0.005 & 0.567 \\
Visual acuity of the better eye (logMAR) & -0.355 & 0.059 & $<0.001^{\dagger}$ \\
Family income & 0.024 & 0.010 & $0.020^{\dagger}$ \\
\hline
\end{tabular}

$\log \mathrm{MAR}=$ logarithm of the minimum angle of resolution.

*Multiple linear regression; ${ }^{\dagger}$ Statistically significant value. 
Conflicts of Interest: None.

Acknowledgements: None.

Funding: None

\section{References}

1. Gerull R, Brauer V, Bassler D, et al. Incidence of retinopathy of prematurity (ROP) and ROP treatment in Switzerland 2006-2015: a population-based analysis. Arch Dis Child Fetal Neonatal Ed 2018;103:F337-42.

2. Ying GS, Bell EF, Donohue P, et al. Perinatal risk factors for the retinopathy of prematurity in postnatal growth and ROP Study. Ophthalmic Epidemiol 2019;26:270-8.

3. Isaza G, Arora S, Bal M, Chaudhary V. Incidence of retinopathy of prematurity and risk factors among premature infants at a neonatal intensive care unit in Canada. $J$ Pediatr Ophthalmol Strabismus 2013;50:27-32.

4. Shah VA, Yeo CL, Ling YL, Ho LY. Incidence, risk factors of retinopathy of prematurity among very low birth weight infants in Singapore. Ann Acad Med Singap 2005;34:169-78.

5. Siswanto JE, Widodo NH, Sauer PJ. Eleven years of retinopathy of prematurity in one neonatal intensive care unit in Jakarta, Indonesia. Arch Dis Child 2018;103:619-21.

6. Choo MM, Martin FJ, Theam LC, U-Teng C. Retinopathy of prematurity in extremely low birth weight infants in Malaysia. J AAPOS 2009;13:446-9.

7. Kang EY, Lien R, Wang NK, et al. Retinopathy of prematurity trends in Taiwan: a 10-year nationwide population study. Invest Ophthalmol Vis Sci 2018;59:3599-607.

8. Agarwal K, Balakrishnan D, Rani PK, Jalali S. Changing patterns of early childhood blinding conditions presenting to a tertiary eye center: the epidemic of retinopathy of prematurity in India. Indian J Ophthalmol 2019;67:816-8.

9. Isayama T. The clinical management and outcomes of extremely preterm infants in Japan: past, present, and future. Transl Pediatr 2019;8:199-211.

10. Hwang JH, Lee EH, Kim EA. Retinopathy of prematurity among very-low-birth-weight infants in Korea: incidence, treatment, and risk factors. J Korean Med Sci 2015;30(Suppl 1):S88-94.

11. Messa AA, Mattos RB, Areco KC, Sallum JM. Vision-related quality of life in children with retinopathy of prematurity. Arq Bras Oftalmol 2015;78:224-8.

12. Kesarwani P, Narang S, Chawla D, et al. Vision-related quality of life in children with treated retinopathy of pre- maturity. Indian J Ophthalmol 2019;67:932-5.

13. Adams GG. ROP in Asia. Eye (Lond) 2020;34:607-8.

14. Petrou S, Krabuanrat N, Khan K. Preference-based health-related quality of life outcomes associated with preterm birth: a systematic review and meta-analysis. Pharmacoeconomics 2020;38:357-73.

15. Birch EE, Cheng CS, Felius J. Validity and reliability of the Children's Visual Function Questionnaire (CVFQ). $J A A$ POS 2007;11:473-9.

16. International Committee for the Classification of Retinopathy of Prematurity. The International Classification of Retinopathy of Prematurity revisited. Arch Ophthalmol 2005;123:991-9.

17. Mills MD. Evaluating the Cryotherapy for Retinopathy of Prematurity Study (CRYO-ROP). Arch Ophthalmol 2007; 125:1276-81.

18. Early Treatment for Retinopathy of Prematurity Cooperative Group. Revised indications for the treatment of retinopathy of prematurity: results of the early treatment for retinopathy of prematurity randomized trial. Arch Ophthalmol 2003;121:1684-94.

19. Aralikatti AK, Mitra A, Denniston AK, et al. Is ethnicity a risk factor for severe retinopathy of prematurity? Arch Dis Child Fetal Neonatal Ed 2010;95:F174-6.

20. Zunaina E, Abdul-Salim I, Siti-Zulaikha-Nashwa MK, et al. Malay premature infants with retinopathy of prematurity: risk factors and screening of NDP gene mutation. $J$ Biomed Clin Sci 2017;2:5-10.

21. Chang KM, Patel DK, Tajunisah I, Subrayan V. The trend of retinopathy of prematurity in Malaysia from 1992 to 2001 based on a nationwide blind schools study. Asia Pac J Public Health 2015;27:217-24.

22. Ozyurt G, Ozyurt A, Ozturk T, et al. Evaluation of maternal attachment, self-efficacy, levels of depression, and anxiety in mothers who have babies diagnosed with retinopathy of prematurity. Ophthalmic Epidemiol 2018;25:140-6.

23. Holditch-Davis D, Miles MS, Weaver MA, et al. Patterns of distress in African-American mothers of preterm infants. $J$ Dev Behav Pediatr 2009;30:193-205.

24. Vijayalakshmi P, Kara T, Gilbert C. Ocular morbidity associated with retinopathy of prematurity in treated and untreated eyes: a review of the literature and data from a tertiary eye-care center in Southern India. Indian Pediatr 2016;53 Suppl 2:S137-42.

25. Scher MS, Johnson MW, Ludington SM, Loparo K. Physiologic brain dysmaturity in late preterm infants. Pediatr Res 2011;70:524-8. 